cornerstone theories of physics and resolving one of the most profound scientific challenges today. "Causality lies at the interface between quantum mechanics and general relativity," says Walther's collaborator Časlav Brukner, a theorist at the Institute for Quantum Optics and Quantum Information in Vienna, "and so it could help us to think about how one could merge the two conceptually."

\section{TANGLES IN TIME}

Causation has been a key issue in quantum mechanics since the mid-1930s, when Einstein challenged the apparent randomness that Niels Bohr and Werner Heisenberg had installed at the heart of the theory. Bohr and Heisenberg's Copenhagen interpretation insisted that the outcome of a quantum measurement - such as checking the orientation of a photon's plane of polarization - is determined at random, and only in the instant that the measurement is made. No reason can be adduced to explain that particular outcome. But in 1935, Einstein and his young colleagues Boris Podolsky and Nathan Rosen (now collectively denoted EPR) described a thought experiment that pushed Bohr's interpretation to a seemingly impossible conclusion.

The EPR experiment involves two particles, $A$ and $B$, that have been prepared with interdependent, or 'entangled', properties. For example, if A has an upward-pointing 'spin' (crudely, a quantum property that can be pictured a little bit like the orientation of a bar magnet), then B must be down, and vice versa.

Both pairs of orientations are possible. But researchers can discover the actual orientation only when they make a measurement on one of the particles. According to the Copenhagen interpretation, that measurement doesn't just reveal the particle's state; it actually fixes it in that instant. That means it also instantly fixes the state of the particle's entangled partner however far away that partner is. But Einstein considered this apparent instant action at a distance impossible, because it would require faster-than-light interaction across space, which is forbidden by his special theory of relativity. Einstein was convinced that this invalidated the Copenhagen interpretation, and that particles A and B must already have welldefined spins before anybody looks at them.

Measurements of entangled particles show, however, that the observed correlation between the spins can't be explained on the basis of preexisting properties. But these correlations don't actually violate relativity because they can't be used to communicate faster than light. Quite how the relationship arises is hard to explain in any intuitive cause-and-effect way.

But what the Copenhagen interpretation does at least seem to retain is a time-ordering logic: a measurement can't induce an effect until after it has been made. For event A to have any effect on event $B, A$ has to happen first. The trouble is that this logic has unravelled over the past decade, as researchers have realized that it is possible to imagine quantum scenarios in which one simply can't say which of two related events happens first.

Classically, this situation sounds impossible. True, we might not actually know whether A or B happened first - but one of them surely did. Quantum indeterminacy, however, isn't a lack of knowledge; it's a fundamental prohibition on pronouncing on any 'true state of affairs' before a measurement is made.

\section{AMBIGUOUS ACTION}

Brukner's group in Vienna, Chiribella's team and others have been pioneering efforts to explore this ambiguous causality in quantum mechanics ${ }^{3,4}$. They have devised ways to create related events $\mathrm{A}$ and $\mathrm{B}$ such that no one can say whether A preceded and led to (in a sense 'caused') B, or vice versa. This arrangement enables information to be shared between $A$ and $B$ in ways that are ruled out if there is a definite causal order. In other words, an indeterminate causal order lets researchers do things with quantum systems that are otherwise impossible.

The trick they use involves creating a special type of quantum 'superposition'. Superpositions of quantum states are well known: a spin, for example, can be placed in a superposition of up and down states. And the two spins in the EPR experiment are in a superposition - in that case involving two particles. It's often said that a quantum object in a superposition exists in two states at once, but more properly it simply cannot be said in advance what the outcome of and 1 , the second qubit experiences a causal superposition of both sequences - meaning there is no defined order to the particle's traversal of the gates (see 'Trippy journeys').

Three years later, Chiribella proposed an explicit experimental procedure for enacting this idea ${ }^{5}$; Walther, Brukner and their colleagues subsequently worked out how to implement it in the lab ${ }^{1}$. The Vienna team uses a series of 'waveplates' (crystals that change a photon's polarization) and partial mirrors that reflect light and also let some pass through. These devices act as the logic gates $\mathrm{A}$ and $\mathrm{B}$ to manipulate the polarization of a test photon. A control qubit determines whether the photon experiences $\mathrm{AB}$ or $\mathrm{BA}$ - or a causal superposition of both. But any attempt to find out whether the photon goes through gate $\mathrm{A}$ or gate $\mathrm{B}$ first will destroy the superposition of gate ordering.

Having demonstrated causal indeterminacy experimentally, the Vienna team wanted to go further. It's one thing to create a quantum superposition of causal states, in which it is simply not determined what caused what (that is, whether the gate order is $\mathrm{AB}$ or $\mathrm{BA}$ ). But the researchers wondered whether it is possible to preserve causal ambiguity even if they spy on the photon as it travels through various gates.

At face value, this would seem to violate the idea that sustaining a superposition depends on not trying to measure it. But researchers are now realizing that in quantum mechanics, it's not exactly what you do that matters, but what you know.

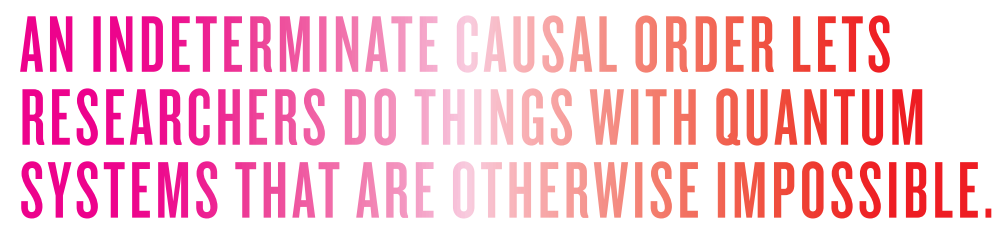

a measurement would be. The two observable states can be used as the binary states ( 1 and 0 ) of quantum bits, or qubits, which are the basic elements of quantum computers.

The researchers extend this concept by creating a causal superposition. In this case, the two states represent sequences of events: a particle goes first through gate $A$ and then through gate B (so that A's output state determines B's input), or vice versa.

In 2009, Chiribella and his co-workers came up with a theoretical way to do an experiment like this using a single qubit as a switch that controls the causal order of events experienced by a particle that acts as second qubit ${ }^{3}$. When the control-switch qubit is in state 0 , the particle goes through gate A first, and then through gate $\mathrm{B}$. When the control qubit is in state 1 , the order of the second qubit is BA. But if that qubit is in a superposition of 0
Last year, Walther and his colleagues devised a way to measure the photon as it passes through the two gates without immediately changing what they know about it ${ }^{6}$. They encode the result of the measurement in the photon itself, but do not read it out at the time. Because the photon goes through the whole circuit before it is detected and the measurement is revealed, that information can't be used to reconstruct the gate order. It's as if you asked someone to keep a record of how they feel during a trip and then relay the information to you later - so that you can't deduce exactly when and where they were when they wrote it down.

As the Vienna researchers showed, this ignorance preserves the causal superposition. "We don't extract any information about the measurement result until the very end of the entire process, when the final readout takes 
place," says Walther. "So the outcome of the measurement process, and the time when it was made, are hidden but still affect the final result."

Other teams have also been creating experimental cases of causal ambiguity by using quantum optics. For example, a group at the University of Waterloo in Canada and the nearby Perimeter Institute for Theoretical Physics has created quantum circuits that manipulate photon states to produce a different causal mash-up. In effect, a photon passes through gates $\mathrm{A}$ and $\mathrm{B}$ in that order, but its state is determined by a mixture of two causal procedures: either the effect of $B$ is determined by the effect of $A$, or the effects of $A$ and $B$ are individually determined by some other event acting on them both, in much the same way that a hot day can increase sunburn cases and ice-cream sales without the two phenomena being directly causally related. As with the Vienna experiments, the Waterloo group found that it's not possible to assign a single causal 'story' to the state the photons acquire . $^{7}$

Some of these experiments are opening up new opportunities for transmitting information. A causal superposition in the order of signals travelling through two gates means that each can be considered to send information to the other simultaneously. "Crudely speaking, you get two operations for the price of one," says Walther. This offers a potentially powerful shortcut for information processing.

Although it has long been known that using quantum superposition and entanglement could exponentially increase the speed of computation, such tricks have previously been played only with classical causal structures. But the simultaneous nature of pathways in a quantum-causal superposition offers a further boost in speed. That potential was apparent when such superpositions were first proposed: quantum theorist Lucien Hardy at the Perimeter Institute ${ }^{8}$ and Chiribella and his co-workers ${ }^{3}$ independently suggested that quantum computers operating with an indefinite causal structure might be more powerful than ones in which causality is fixed.

Last year, Brukner and his co-workers showed $^{9}$ that building such a shortcut into an information-processing protocol with many gates should give an exponential increase in the efficiency of communication between gates, which could be beneficial for computation. "We haven't reached the end yet of the possible speed-ups," says Brukner. "Quantum mechanics allows way more."

\section{TRIPPY JOURNEYS}

At the quantum level, the normal rules of cause and effect need not apply. To demonstrate this, researchers have sent photons through a series of gates with an ambiguous causal order. The set-up is analogous to a train's ath through a pair of gates governed by a two-way switch.

The switch in this case is a quantum bit, or qubit, which selects the route that the train takes.

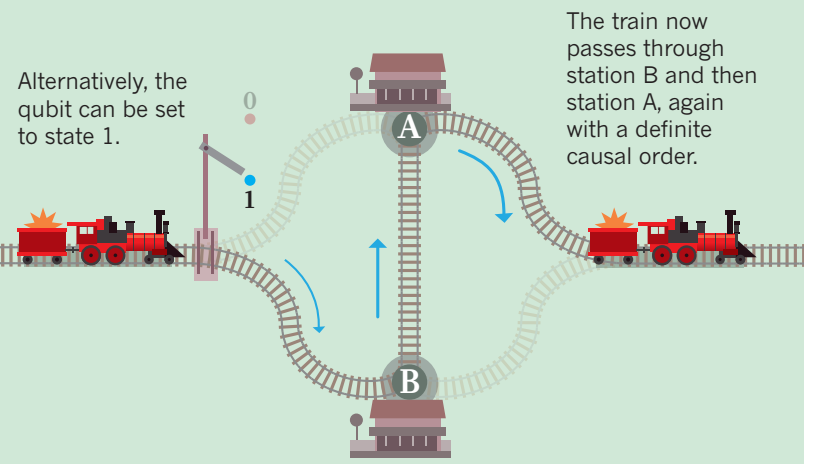

If the qubit is set to a superposition of 1 and 0 , it is impossible to say whether the train goes through A or B first.
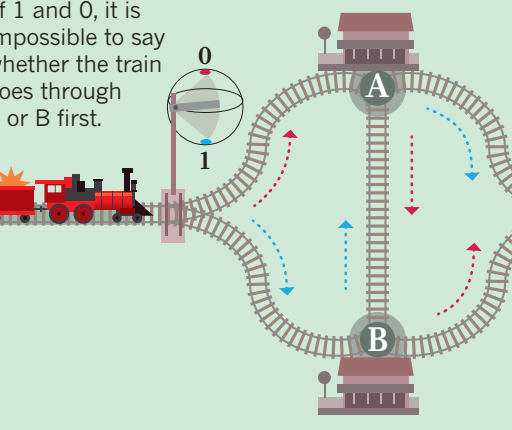

The system has an ambiguous sequence in time: an indefinite causal order.

If the qubit switch is set to state 0 , the train passes through station $B$. This route has a definite causal order.

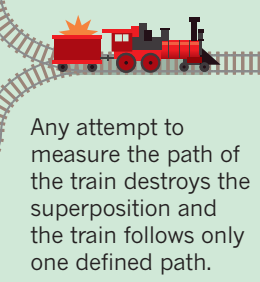

two decades, some physicists and mathematicians, including Hardy ${ }^{10}$ and Brukner ${ }^{11}$, have sought to clarify things by building 'quantum reconstructions': attempts to derive at least some characteristic properties of quantum-mechanical systems such as entanglement and superpositions - from simple axioms about, say, what can and can't be done with the information encoded in the states (see Nature 501, 154-156; 2013).

"The framework of causal models provides a new perspective on these questions," says Katja Ried, a physicist at the University of Innsbruck in Austria who previously worked with the University of Waterloo team on developing systems with causal ambiguity. "If quantum theory is a theory about how nature processes and distributes information, then asking in which ways events can influence each other may reveal the rules of this processing."

And quantum causality might go even further by showing how one can start to fit quantum theory into the framework of general relativity, which accounts for gravitation. "The fact that causal structure plays such a central role in general relativity motivates us to investigate in which ways it can 'behave quantumly", says Ried.

"Most of the attempts to understand quantum mechanics involve trying to save some aspects of the old classical picture, such as particle trajectories," says Brukner. But history shows us that what is generally needed in such cases is something more, he says - something that goes beyond the old ideas, such as a new way of thinking about causality itself. "When you have a radical theory, to understand it you usually need something even more radical."
It's not terribly complicated to build the necessary quantum-circuit architectures, either - you just need quantum switches similar to those Walther has used. "I think this could find applications soon," Brukner says.

\section{UNITY IN THE UNIVERSE}

The bigger goal, however, is theoretical. Quantum causality might supply a point of entry to some of the hardest questions in physics - such as where quantum mechanics comes from.

Quantum theory has always looked a little ad hoc. The Schrödinger equation works marvellously to predict the outcomes of quantum experiments, but researchers are still arguing about what it means, because it's not clear what the physics behind it is. Over the past

\section{Philip Ball is a freelance writer in London.}

1. Procopio, L. M. et al. Nature Commun. 6, 7913 (2015).

2. Brukner, Č. Nature Phys. 10, 259-263 (2014).

3. Chiribella, G., D'Ariano, G. M., Perinotti, P. \& Valiron, B. Phys. Rev. A 88, 022318 (2013).

4. Oreshkov, O., Costa, F. \& Brukner, Č. Nature Commun. 3, 1092 (2012).

5. Chiribella, G. Phys. Rev. A 86, 040301(R) (2012).

6. Rubino, G. et al. Sci. Adv. 3, e1602589 (2017).

7. MacLean, J.-P. W., Ried, K., Spekkens, R. W. \& Resch, K. J. Nature Commun. 8, 15149 (2017)

8. Hardy, L. Preprint at http://arxiv.org/abs/ quant-ph/0701019 (2007).

9. Allard Guérin, P., Feix, A., Araújo, M. \& Brukner, Č. Phys. Rev. Lett. 117, 100502 (2016).

10. Hardy, L. Preprint at http://arxiv.org/abs/ quant-ph/0101012 (2001).

11.Dakić, B. \& Brukner, Č. in Deep Beauty: Understanding the Quantum World through Mathematical Innovation (ed. Halvorson, $\mathrm{H}$.) 365-392 (Cambridge Univ. Press, 2011). 